J. Korean Math. Soc. 49 (2012), No. 1, pp. 31-47

http://dx.doi.org/10.4134/JKMS.2012.49.1.031

\title{
HOMOLOGICAL PROPERTIES OF MODULES OVER DING-CHEN RINGS
}

\author{
GANG YANG
}

\begin{abstract}
The so-called Ding-Chen ring is an $n$-FC ring which is both left and right coherent, and has both left and right self FP-injective dimensions at most $n$ for some non-negative integer $n$. In this paper, we investigate the classes of the so-called Ding projective, Ding injective and Gorenstein flat modules and show that some homological properties of modules over Gorenstein rings can be generalized to the modules over Ding-Chen rings. We first consider Gorenstein flat and Ding injective dimensions of modules together with Ding injective precovers. We then discuss balance of functors Hom and tensor.
\end{abstract}

\section{Introduction and preliminaries}

Non-commutative Gorenstein rings were defined and studied by Iwanaga in [21] and [22]. Later Enochs and Jenda defined and studied the so-called Gorenstein projective, Gorenstein injective and Gorenstein flat modules and developed Gorenstein homological algebra [14] on modules over such rings. Those modules over general associative rings have also been studied by Holm in [18] and [19]. The point of this paper is to describe how the homological theory on modules over a Gorenstein ring generalizes to a homological theory on modules over a so-called Ding-Chen ring.

In [26], Stenström introduced the notion of an FP-injective module and studied FP-injective modules over coherent rings. An $R$-module $E$ is called $F P$ injective if $\operatorname{Ext}_{R}^{1}(A, E)=0$ for all finitely presented $R$-modules $A$. More generally, the $F P$-injective dimension of an $R$-module $B$ is defined to be the least integer $n \geq 0$ such that $\operatorname{Ext}_{R}^{n+1}(A, B)=0$ for all finitely presented $R$-modules $A$. The FP-injective dimension of $B$ is denoted $\mathrm{FP}-\mathrm{id}_{R}(B)$ and equals $\infty$ if no such $n$ above exists. $F C$ rings, as the coherent version of quasi-Frobenius rings where Noetherian is replaced by coherent and self injective is replaced by self FP-injective, were introduced by Damiano in [3]. Just as Gorenstein rings

Received July 22, 2010; Revised January 10, 2011.

2010 Mathematics Subject Classification. 16E05, 16E65, 18G20, 18G25.

Key words and phrases. Ding-Chen ring, Ding projective and Ding injective module, Gorenstein flat module, precover, preenvelope, balanced functors.

This work was partly supported by the NSF of China (Grants 11101197 and 10901129). 
are natural generalizations of quasi-Frobenius rings, Ding and Chen extended FC rings to $n$-FC rings $[4,6]$ which are seen to have many properties similar to those of $n$-Gorenstein rings. Just as a ring is called Gorenstein when it is $n$-Gorenstein for some non-negative integer $n$ (a ring $R$ is called $n$-Gorenstein if it is a left and right Noetherian ring with self injective dimension at most $n$ on both sides for some non-negative integer $n$ ), Gillespie first called a ring Ding-Chen when it is $n$-FC for some $n$ [17].

Definition 1.1. A ring $R$ is called an $n-F C$ ring if it is both left and right coherent and $\mathrm{FP}$-id $\left({ }_{R} R\right)$ and $\mathrm{FP}-\mathrm{id}\left(R_{R}\right)$ are both less than or equal to $n$. $\mathrm{A}$ 0 -FC ring is just called FC. A ring $R$ is called Ding-Chen if it is an $n$-FC ring for some non-negative integer $n$.

Recall from [14] that an $R$-module $M$ is Gorenstein projective if there exists an exact sequence $\cdots \longrightarrow P_{1} \longrightarrow P_{0} \longrightarrow P^{0} \longrightarrow P^{1} \longrightarrow \cdots$ of projective $R$-modules with $M=\operatorname{Ker}\left(P^{0} \longrightarrow P^{1}\right)$ such that $\operatorname{Hom}(-, P)$ leaves the sequence exact whenever $P$ is a projective $R$-module. An $R$-module $N$ is called Gorenstein injective if there exists an exact sequence $\cdots \longrightarrow I_{1} \longrightarrow I_{0} \longrightarrow$ $I^{0} \longrightarrow I^{1} \longrightarrow \cdots$ of injective $R$-modules with $N=\operatorname{Ker}\left(I^{0} \longrightarrow I^{1}\right)$ such that $\operatorname{Hom}(I,-)$ leaves the sequence exact whenever $I$ is an injective $R$-module. As special cases of the Gorenstein projective and Gorenstein injective modules, the Ding projective and Ding injective modules were introduced and studied by Ding, Mao and co-authors in [24] and [7] as strongly Gorenstein flat and Gorenstein FP-injective modules respectively. These two classes of modules over coherent rings possess many nice properties analogous to those of Gorenstein projective and Gorenstein injective modules over Noetherian rings. For example, when $R$ is an $n$-Gorenstein ring, Hovey [20] showed that the category of $R$-modules has two Quillen equivalent model structures, i.e., the Gorenstein projective model structure and the Gorenstein injective model structure. Similar results were shown by Gillespie in [17] when $R$ is an $n$-FC ring.

Definition $1.2([7])$. An $R$-module $M$ is called Ding projective if there exists an exact sequence of projective $R$-modules

$$
\cdots \longrightarrow P_{1} \longrightarrow P_{0} \longrightarrow P^{0} \longrightarrow P^{1} \longrightarrow \cdots
$$

with $M=\operatorname{Ker}\left(P^{0} \longrightarrow P^{1}\right)$ and which remains exact after applying $\operatorname{Hom}(-, F)$ for any flat $R$-module $F$.

Definition $1.3([24])$. An $R$-module $N$ is called Ding injective if there exists an exact sequence of injective $R$-modules

$$
\cdots \longrightarrow I_{1} \longrightarrow I_{0} \longrightarrow I^{0} \longrightarrow I^{1} \longrightarrow \cdots
$$

with $N=\operatorname{Ker}\left(I^{0} \longrightarrow I^{1}\right)$ and which remains exact after applying $\operatorname{Hom}(E,-)$ for any FP-injective $R$-module $E$. 
Note that every Ding injective (respectively, Ding projective) $R$-module $N$ is Gorenstein injective (respectively, Gorenstein projective), and if $R$ is Gorenstein, then every Gorenstein injective $R$-module is Ding injective (respectively, Gorenstein projective) [17].

Definition 1.4 ([16]). An $R$-module $M$ is called Gorenstein flat if there exists an exact sequence of flat $R$-modules

$$
\cdots \longrightarrow F_{1} \longrightarrow F_{0} \longrightarrow F^{0} \longrightarrow F^{1} \longrightarrow \cdots
$$

with $M=\operatorname{Ker}\left(F^{0} \longrightarrow F^{1}\right)$ and which remains exact after applying $I \otimes_{R}-$ for any injective right $R$-module $I$.

In [24], Mao and Ding showed that if a left $R$-module $M$ is Gorenstein flat, then $M^{+}=\operatorname{Hom}_{\mathbb{Z}}(M, \mathbb{Q} / \mathbb{Z})$ is a Ding injective right $R$-module, and the converse holds when $R$ is a right coherent ring. By [7, Proposition 2.3] (also [17, Proposition 3.12]), if $R$ is a right coherent ring, then any Ding projective left $R$-module $M$ is Gorenstein flat. We refer the reader to [6, Theorem 5] for characterizations of Gorenstein flat modules over Ding-Chen rings.

Given a class $\mathcal{H}$ of $R$-modules, we will denote the class of $R$-modules $X$ satisfying $\operatorname{Ext}^{1}(H, X)=0$ (respectively, $\operatorname{Ext}^{1}(X, H)=0$ ) for every $H \in \mathcal{H}$ by $\mathcal{H}^{\perp}$ (respectively, ${ }^{\perp} \mathcal{H}$ ). Following [14], a pair of classes of $R$-modules $(\mathcal{A}, \mathcal{B})$ is said to be a cotorsion pair if $\mathcal{A}^{\perp}=\mathcal{B}$ and ${ }^{\perp} \mathcal{B}=\mathcal{A}$. A cotorsion pair $(\mathcal{A}, \mathcal{B})$ is said to be complete if for any $R$-module $X$ there are exact sequences $0 \rightarrow X \rightarrow B \rightarrow A \rightarrow 0$ and $0 \rightarrow \widetilde{B} \rightarrow \widetilde{A} \rightarrow X \rightarrow 0$ respectively with $B, \widetilde{B} \in \mathcal{B}$ and $A, \widetilde{A} \in \mathcal{A}$.

Let $\mathcal{C}$ be a class of $R$-modules and $X$ an $R$-module. Following [9], we say that a homomorphism $f: C \longrightarrow X$ is a $\mathcal{C}$-precover if $C \in \mathcal{C}$ and the abelian group homomorphism $\operatorname{Hom}_{R}\left(C^{\prime}, f\right): \operatorname{Hom}_{R}\left(C^{\prime}, C\right) \rightarrow \operatorname{Hom}_{R}\left(C^{\prime}, X\right)$ is surjective for each $C^{\prime} \in \mathcal{C}$. A $\mathcal{C}$-precover $f: C \longrightarrow X$ is called a $\mathcal{C}$-cover if every endomorphism $g: C \rightarrow C$ such that $f g=f$ is an isomorphism. Dually we have the definitions of a $\mathcal{C}$ - $($ pre $)$ envelope. $\mathcal{C}$-covers $(\mathcal{C}$-envelopes $)$ may not exist in general, but if they exist, they are unique up to isomorphism.

Throughout we assume all rings have an identity and all modules are unitary. Unless stated otherwise, an $R$-module will be understood to be a left $R$-module. We denote the flat dimension of an $R$-module $M$ by $\mathrm{fd}_{R}(M)$. The character module $\operatorname{Hom}_{\mathbb{Z}}(M, \mathbb{Q} / \mathbb{Z})$ of the module $M$ is denoted by $M^{+}$.

\section{Ding injectivity and Gorenstein flatness of modules}

In this section, we first study the existence of Ding injective precovers of certain modules and then the duality properties between Ding injective and Gorenstein flat modules.

Recall that a short exact sequence, $0 \rightarrow A \rightarrow B \rightarrow C \rightarrow 0$, of $R$-modules is called pure exact if $0 \rightarrow M \otimes_{R} A \rightarrow M \otimes_{R} B \rightarrow M \otimes_{R} C \rightarrow 0$ is exact for every right $R$-module $M$. A submodule $A$ of an $R$-module $B$ is said to be a pure 
submodule if $0 \rightarrow A \rightarrow B \rightarrow C \rightarrow 0$ is pure exact. A module $X$ is called pure injective if the sequence $0 \rightarrow \operatorname{Hom}_{R}(C, X) \rightarrow \operatorname{Hom}_{R}(B, X) \rightarrow \operatorname{Hom}_{R}(A, X) \rightarrow$ 0 is exact for every pure exact sequence $0 \rightarrow A \rightarrow B \rightarrow C \rightarrow 0$.

The following result was proved in [24, Theorem 4.1] under an extra assumption that $R$ is a perfect ring.

Theorem 2.1. Let $R$ be a Ding-Chen ring. Then every pure injective left $R$-module $N$ has a Ding injective precover.

Proof. Since every right module over a Ding-Chen ring $R$ has a Gorenstein flat preenvelope by [24, Theorem 5.3], so has $N^{+}$. Let $f: N^{+} \rightarrow G$ be a Gorenstein flat preenvelope of $N^{+}$. Then, by [24, Lemma 2.8], [6, Proposition 12] and [11, Corollary 3.2], $f^{+}: G^{+} \rightarrow N^{++}$is a Ding injective precover of $N^{++}$. On the other hand, $N$ is a direct summand of $N^{++}$by [27, Proposition 2.3.5] since $N$ is pure injective, and then it is easy to see that $\pi f^{+}: G^{+} \rightarrow N$ is a Ding injective precover of $N$, where $\pi: N^{++} \rightarrow N$ is the canonical projection. This completes the proof.

According to [24, Lemma 2.8], if $R$ is left coherent, then a right $R$-module $M$ is Gorenstein flat if and only if $M^{+}$is Ding injective. Moreover, if $R$ is a Ding-Chen ring, then $N^{+}$is a Gorenstein flat right $R$-module whenever $N$ is a Ding injective left $R$-module (see [6, Proposition 12]).

Proposition 2.2. Let $R$ be a Ding-Chen ring and $N$ be a pure injective left $R$-module. If $N^{+}$is a Gorenstein flat right $R$-module, then $N$ is Ding injective.

Proof. By [24, Lemma 2.8], $N^{++}$is Ding injective. Since $N$ is a pure injective left $R$-module, $N$ is a direct summand of $N^{++}$by [27, Proposition 2.3.5]. Thus it follows from [24, Proposition 2.6] that $N$ is Ding injective.

Note that $\mathrm{Xu}$ proved in [27] that for a commutative ring $R$, a simple $R$ module is flat if and only if it is injective. Furthermore, Ding and Chen proved in [5] that if $R$ is a commutative coherent ring, then the flat dimension and the injective dimension of any simple $R$-module are identical. In the following, we focus on Gorenstein flat and Ding injective dimensions of some special modules over Ding-Chen rings.

Definition 2.3. Given a left $R$-module $M$. Let $1 . \operatorname{Did}_{R}(M)\left(\operatorname{Did}_{R}(M)\right.$ for short) denote $\inf \left\{n \mid\right.$ there exists an exact sequence $0 \rightarrow M \rightarrow E_{0} \rightarrow E_{1} \rightarrow$ $\cdots \rightarrow E_{n} \rightarrow 0$ with $E_{i}$ Ding injective for any $\left.0 \leq i \leq n\right\}$ and call $1 . \operatorname{Did}_{R}(M)$ the Ding injective dimension of $M$. If no such $n$ exists, set $1 . \operatorname{Did}_{R}(M)=\infty$.

In a dual way, the Ding projective dimension, $\operatorname{Dpd}_{R}(M)$, of an $R$-module $M$ can be defined.

Definition 2.4. Given a right $R$-module $N$. The Gorenstein flat dimension of $N$, denoted by $\operatorname{r.Gfd} \operatorname{Gf}_{R}(N)\left(\operatorname{Gfd}_{R}(N)\right.$ for short $)$, is defined as $\inf \{n \mid$ there exists an exact sequence $0 \rightarrow G_{n} \rightarrow \cdots \rightarrow G_{1} \rightarrow G_{0} \rightarrow N \rightarrow 0$ with $G_{i}$ Gorenstein flat for any $0 \leq i \leq n\}$. If no such $n$ exists, set $\operatorname{r} \cdot \operatorname{Gfd}_{R}(M)=\infty$. 
The Gorenstein flat dimension refines the classical flat dimension (see [14] and $[18])$.

Lemma 2.5. Let $R$ be a Ding-Chen ring and $n$ a fixed non-negative integer. Then the following statements are equivalent for a left $R$-module $M$ :

(1) $\operatorname{l.Did}_{R}(M) \leq n$.

(2) $\operatorname{Ext}_{R}^{i}(J, M)=0$ for any FP-injective $R$-module $J$ and any $i \geq n+1$.

Proof. The proof is dual to that of [7, Lemma 3.4].

Lemma 2.6. Let $R$ be a Ding-Chen ring and $n$ a fixed non-negative integer. Then the following statements are equivalent for a right $R$-module $N$ :

(1) $\mathrm{r} \cdot \operatorname{Gfd}_{R}(N) \leq n$.

(2) $\operatorname{Tor}_{i}^{R}(N, J)=0$ for any FP-injective left $R$-module $J$ and any $i \geq n+1$.

Proof. $(2) \Rightarrow(1)$ By [6, Theorem 7], we get that $r . \operatorname{Gfd}_{R}(M)<\infty$. Then the result follows from $[2$, Theorem 2.8] since any injective module is FP-injective.

$(1) \Rightarrow(2)$ Let $0 \rightarrow K_{n} \rightarrow F_{n-1} \rightarrow \cdots \rightarrow F_{1} \rightarrow F_{0} \rightarrow M \rightarrow 0$ be an exact sequence of right $R$-modules, where each $F_{i}$ is flat. Then, by [2, Lemma 2.9], we get that $K_{n}$ is Gorenstein flat. Hence if $J$ is any FP-injective left $R$-module, then $\operatorname{Tor}_{n+i}^{R}(M, J) \cong \operatorname{Tor}_{i}^{R}\left(K_{n}, J\right)=0$ for any $i \geq 1$ by [6, Theorem 5], as desired.

Lemma 2.7 ([25, Lemma 3.1]). Let $R$ be any ring and $I$ be an ideal of $R$ such that $R / I$ is a semi-simple ring. Then for any left $R$-module $X$ and a positive integer $n, \operatorname{Ext}_{R}^{n}(X, R / I)=0$ if and only if $\operatorname{Tor}_{n}^{R}(R / I, X)=0$.

The following result generalizes [25, Theorem 3.2] which gives a characterization of Gorenstein flat dimension of related modules over Gorenstein rings.

Theorem 2.8. Let $R$ be a Ding-Chen ring and $I$ be an ideal of $R$ such that $R / I$ is a semi-simple ring. Then $\mathrm{r} \cdot \operatorname{Gfd}_{R}(R / I)=1 \cdot \operatorname{Did}_{R}(R / I)$.

Proof. Use Lemmas 2.5, 2.6 and 2.7.

Remark 2.9. It was proved by Enochs and Jenda that every $R$-module has finite Gorenstein injective (respectively, Gorenstein flat) dimension whenever $R$ is Gorenstein [14, Theorem 12.3.1]. It was also proved by Ding and Chen that every $R$-module has finite Gorenstein flat dimension whenever $R$ is DingChen [6, Theorem 7]. In [24], Mao and Ding showed that if $R$ is Ding-Chen and left perfect, then every $R$-module has finite Ding injective dimension [24, Lemma 3.1]. But it is still not known whether or not every $R$-module has finite Ding injective dimension when $R$ is just a Ding-Chen ring.

Corollary 2.10. Let $R$ be a Ding-Chen ring and $I$ be an ideal of $R$ such that $R / I$ is a semi-simple ring. Then $\operatorname{l.Did}_{R}(R / I)<\infty$.

Proof. It follows from Theorem 2.8 and Remark 2.9. 
Recall that $R$ is called a semi-local ring if $R / J(R)$ is a semi-simple ring, where $J(R)$ denotes the Jacobson radical of $R$. By Theorem 2.8, we immediately have the following result.

Corollary 2.11. Let $R$ be a Ding-Chen ring. If $R$ is a semi-local ring, then $\operatorname{r.Gfd}_{R}(R / J(R))=1 . \operatorname{Did}_{R}(R / J(R))$.

Corollary 2.12. Let $R$ be a Ding-Chen ring. If $R$ is a commutative ring, then for any simple $R$-module $T, \operatorname{Gfd}_{R}(T)=\operatorname{Did}_{R}(T)$. In particular, $T$ is Gorenstein flat if and only if it is Ding injective.

Proof. Let $T$ be a simple $R$-module. Then there exists a maximal ideal $\mathfrak{M}$ of $R$ such that $T \cong R / \mathfrak{M}$. So $T$ is a simple ring and hence the assertion follows from Theorem 2.8 .

Corollary 2.13. Let $R$ be a commutative Ding-Chen ring. Then any simple $R$-module has finite Ding injective dimension.

Proof. It follows from Remark 2.9 and Corollary 2.12.

The following result generalizes [25, Theorem 3.5] which is proved for Gorenstein rings.

Theorem 2.14. Let $R$ be a Ding-Chen ring and $R \rightarrow S$ be a homomorphism of rings. If ${ }_{S} E$ is an injective cogenerator for the category of left $S$-modules, then ${\mathrm{r} . G^{-}}_{R}(S)=1 . \operatorname{Did}_{R}(E)$.

Proof. It is trivial that $S$ can be regarded as a bimodule ${ }_{S} S_{R}$, and ${ }_{S} E$ can be regarded as a left $R$-module.

Let $J$ be any FP-injective left $R$-module. Then for any $n \geq 1$, we get that $\operatorname{Ext}_{R}^{n}(J, E) \cong \operatorname{Hom}_{S}\left(\operatorname{Tor}_{n}^{R}(S, J), E\right)$ since

$$
\operatorname{Ext}_{R}^{n}\left(J, \operatorname{Hom}_{S}(S, E)\right) \cong \operatorname{Hom}_{S}\left(\operatorname{Tor}_{n}^{R}(S, J), E\right) .
$$

Because ${ }_{S} E$ is an injective cogenerator for the category of left $S$-modules, $\operatorname{Ext}_{R}^{n}(J, E)=0$ if and only if $\operatorname{Tor}_{n}^{R}(S, J)=0$. Thus the result follows easily from Lemmas 2.5 and 2.6.

Finally, we give an application of Theorem 2.14.

Corollary 2.15. Let $R$ be a Ding-Chen ring and $R \rightarrow S$ be a homomorphism of rings. Then $\operatorname{r.} \operatorname{Gfd}_{R}(S)=1 \cdot \operatorname{Did}_{R}\left(S^{+}\right)$.

Proof. It follows easily from Theorem 2.14 since $S^{+}$is an injective cogenerator for the category of left $S$-modules.

\section{Balanced functors}

In this section, we investigate the balanced functors due to Enochs and Jenda $[12,13,14]$. We recall that if $\mathcal{X}, \mathcal{Y}, \mathcal{B}$ and $\mathcal{E}$ are classes of objects of an abelian 
category $\mathcal{A}$, then we say $\operatorname{Hom}(-,-)$ is right balanced on $\mathcal{X} \times \mathcal{Y}$ by $\mathcal{B} \times \mathcal{E}$ if for any objects $X \in \mathcal{X}$ and $Y \in \mathcal{Y}$ there exist complexes

$$
\cdots \rightarrow B_{2} \rightarrow B_{1} \rightarrow B_{0} \rightarrow X \rightarrow 0
$$

and

$$
0 \rightarrow Y \rightarrow E^{0} \rightarrow E^{1} \rightarrow E^{2} \rightarrow \cdots
$$

such that $B_{i} \in \mathcal{B}, E^{i} \in \mathcal{E}$ for $i \geq 0$ and such that $\operatorname{Hom}(-, E)$ makes the first complex exact whenever $E \in \mathcal{E}$ and such that $\operatorname{Hom}(B,-)$ makes the second complex exact whenever $B \in \mathcal{B}$.

The definition above is easily modified to give the definition of a left or a right balanced functor $-\otimes-$ (see $[12,13,14]$ for more details $)$. We will let $\mathcal{D} \mathcal{P}$, $\mathcal{D I}, \mathcal{G F}$ denote the classes of Ding projective, Ding injective, and Gorenstein flat modules, respectively. The category of left (respectively, right) $R$-modules is denoted by ${ }_{R} \mathcal{M}$ (respectively, $\mathcal{M}_{R}$ ).

We begin with the following result.

Proposition 3.1 ([17, Theorem 4.2]). Let $R$ be an $n-F C$ ring and $M$ an $R$ module. Then the following are equivalent:

(1) $\operatorname{fd}_{R}(M)<\infty$.

(2) $\operatorname{fd}_{R}(M) \leq n$.

(3) $\mathrm{FP}_{R}-\mathrm{id}_{R}(M)<\infty$.

(4) $\mathrm{FP}_{-i d}(M) \leq n$.

By the above, we see that for a Ding-Chen ring $R$, the class of all modules with finite flat dimension and the class of all modules with finite FP-injective dimension are the same, and we use $\mathcal{W}$ to denote this class throughout this section. Ding and Mao proved that $\left({ }^{\perp} \mathcal{W}, \mathcal{W}\right)$ forms a complete cotorsion pair when $R$ is a Ding-Chen ring [8, Theorem 3.8]. Also, $\left(\mathcal{W}, \mathcal{W}^{\perp}\right)$ forms a complete cotorsion pair when $R$ is a Ding-Chen ring [23, Theorem 3.4]. Moreover, Gillespie proved that an $R$-module $M$ is Ding projective if and only if $M \in{ }^{\perp} \mathcal{W}$, an $R$-module $N$ is Ding injective if and only if $N \in \mathcal{W}^{\perp}[17$, Corollaries 4.5 and 4.6].

Lemma 3.2. Let $R$ be a Ding-Chen ring. Then the following statements hold:

(1) For any $R$-module $X$, there exists an exact sequence $0 \rightarrow K \rightarrow D \rightarrow$ $X \rightarrow 0$ such that $D \rightarrow X$ is a Ding projective precover of $X$, and $K \in \mathcal{W}$.

(2) For any $R$-module $Y$, there exists an exact sequence $0 \rightarrow Y \rightarrow C \rightarrow$ $N \rightarrow 0$ such that $Y \rightarrow C$ is a Ding injective preenvelope of $Y$, and $N \in \mathcal{W}$.

Proof. Easy.

Definition 3.3. Let $\mathcal{A}$ be a class of $R$-modules. A complex $\mathbf{A}=\cdots \rightarrow A_{2} \rightarrow$ $A_{1} \rightarrow A_{0} \rightarrow X \rightarrow 0$ is called a proper left $\mathcal{A}$-resolution of $X$ if each $A_{i} \in \mathcal{A}$ and if $A_{0} \rightarrow X, A_{i+1} \rightarrow \operatorname{Ker}\left(A_{i} \rightarrow A_{i-1}\right)$ for $i \geq 0$ are all $\mathcal{A}$-precovers (equivalently, whenever $A \in \mathcal{A}$, the sequence $\operatorname{Hom}_{R}(A, \mathbf{A})=\cdots \rightarrow \operatorname{Hom}_{R}\left(A, A_{2}\right) \rightarrow$ $\operatorname{Hom}_{R}\left(A, A_{1}\right) \rightarrow \operatorname{Hom}_{R}\left(A, A_{0}\right) \rightarrow \operatorname{Hom}_{R}(A, X) \rightarrow 0$ is exact), where $A_{-1}=X$. 
In a dual way, a proper right $\mathcal{A}$-resolution $\mathbf{A}^{\prime}=0 \rightarrow Y \rightarrow A^{0} \rightarrow A^{1} \rightarrow$ $A^{2} \rightarrow \cdots$ of $Y$ can be defined.

From Lemma 3.2 above we get that if $R$ is Ding-Chen, then every $R$-module $X$ admits a proper left Ding projective resolution and a proper right Ding injective resolution.

Lemma 3.4. Assume that $R$ is a Ding-Chen ring, $X$ is an $R$-module, and let $\mathbf{D}=\cdots \rightarrow D_{1} \rightarrow D_{0} \rightarrow X \rightarrow 0$ be a proper left Ding projective resolution of $X$. Then $\operatorname{Hom}_{R}(\mathbf{D}, C)$ is exact for all Ding injective $R$-modules $C$.

Proof. We split the proper left Ding projective resolution $\mathbf{D}$ (which is clearly seen exact since projective $R$-modules are Ding projective) into short exact sequences. Hence it suffices to show exactness of $\operatorname{Hom}_{R}(\mathbf{T}, C)$ for all Ding injective $R$-modules $C$ and all short exact sequences

$$
\mathbf{T}=0 \rightarrow L \rightarrow D \rightarrow X \rightarrow 0,
$$

where $D \rightarrow X$ is a Ding projective precover of $X$ (recall that Ding projective precovers are always surjective). By Lemma 3.2, there is a special short exact sequence,

$$
\mathbf{T}^{\prime}=0 \longrightarrow L^{\prime} \stackrel{\iota}{\longrightarrow} D^{\prime} \stackrel{\pi}{\longrightarrow} X \longrightarrow 0
$$

where $\pi: D^{\prime} \rightarrow X$ is a Ding projective precover and $\operatorname{fd}_{R}\left(L^{\prime}\right)<\infty$.

It is easy to see that the complexes $\mathbf{T}$ and $\mathbf{T}^{\prime}$ are homotopically equivalent, and thus so are the complexes $\operatorname{Hom}_{R}(\mathbf{T}, C)$ and $\operatorname{Hom}_{R}\left(\mathbf{T}^{\prime}, C\right)$ for every (Ding injective) $R$-module $C$. Hence it suffices to show the exactness of $\operatorname{Hom}_{R}\left(\mathbf{T}^{\prime}, C\right)$ whenever $C$ is Ding injective.

Now let $C$ be any Ding injective $R$-module. We need to prove the exactness of

$$
\operatorname{Hom}_{R}\left(D^{\prime}, C\right) \stackrel{\operatorname{Hom}_{\mathrm{R}}(\iota, \mathrm{C})}{\longrightarrow} \operatorname{Hom}_{R}\left(L^{\prime}, C\right) \longrightarrow 0 .
$$

To show this, let $f: L^{\prime} \rightarrow C$ be any homomorphism. We wish to find $g: D^{\prime} \rightarrow$ $C$ such that $g \iota=f$. Now pick an exact sequence

$$
0 \longrightarrow \widetilde{C} \longrightarrow I \stackrel{\alpha}{\longrightarrow} C \longrightarrow 0,
$$

where $I$ is injective, and $\widetilde{C}$ is Ding injective. Since $\widetilde{C}$ is Ding injective and $\operatorname{fd}_{R}\left(L^{\prime}\right)<\infty$, we get $\operatorname{Ext}_{R}^{1}\left(L^{\prime}, \widetilde{C}\right)=0$ by [17, Corollary 4.5], and thus a lifting $\mu: L^{\prime} \rightarrow I$ with $\alpha \mu=f:$

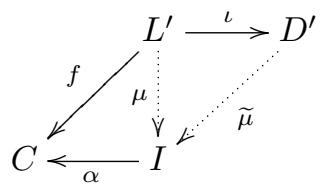

Next, injectivity of $I$ gives $\widetilde{\mu}: D^{\prime} \rightarrow I$ with $\widetilde{\mu} \iota=\mu$. Now $g=\alpha \widetilde{\mu}: D^{\prime} \rightarrow C$ is the desired map.

With a similar proof we get: 
Lemma 3.5. Assume that $R$ is a Ding-Chen ring, $Y$ is an $R$-module, and let $\mathbf{C}=0 \rightarrow Y \rightarrow C^{0} \rightarrow C^{1} \rightarrow \cdots$ be a proper right Ding injective resolution of $Y$. Then $\operatorname{Hom}_{R}(D, \mathbf{C})$ is exact for all Ding projective $R$-modules $D$.

It was proved by Enochs and Jenda [14, Theorem 12.1.4] that the functor $\operatorname{Hom}_{R}(-,-)$ is right balanced on ${ }_{R} \mathcal{M} \times{ }_{R} \mathcal{M}$ by $\mathcal{G}$ or Proj $\times \mathcal{G}$ or $\mathcal{I} n j$ when $R$ is Gorenstein, where $\mathcal{G}$ orP $\operatorname{Proj}$ denotes the class of Gorenstein projective $R$-modules, and $\mathcal{G}$ or $\mathcal{I} n j$ the class of Gorenstein injective $R$-modules. Later, Holm generalized the result as [19, Theorem 3.6] which implies that over any associative ring $R$, the functor $\operatorname{Hom}_{R}(-,-)$ is right balanced on $\mathcal{V} \times \mathcal{W}$ by $\mathcal{G}$ or $\operatorname{Proj} \times \mathcal{G}$ or $\mathcal{I} n j$, where $\mathcal{V}$ denotes the class of $R$-modules with finite Gorenstein projective dimension, and $\mathcal{W}$ the class of $R$-modules with finite Gorenstein injective dimension.

Though we don't know whether or not every $R$-module has finite Ding injective (or projective) dimension when $R$ is a Ding-Chen ring (see [7, Theorem 3.6] and [24, Lemma 3.1] for partial answer), the two lemmas above give the following result.

Theorem 3.6. Let $R$ be a Ding-Chen ring. Then $\operatorname{Hom}_{R}(-,-)$ is right balanced on ${ }_{R} \mathcal{M} \times{ }_{R} \mathcal{M}$ by $\mathcal{D P} \times \mathcal{D I}$.

Remark 3.7. By Theorem 3.6 together with [14, Theorem 8.2.14], if $R$ is DingChen, then we can compute right derived functors of $\operatorname{Hom}_{R}(X, Y)$ using either a proper left Ding projective resolution of $X$ or a proper right Ding injective resolution of $Y$. We will denote these derived functors by $\operatorname{Dext}_{R}^{i}(X, Y)$. Then it is easy to check the following properties of $\operatorname{Dext}_{R}^{i}$ :

(1) $\operatorname{Dext}_{R}^{0}(-,-)=\operatorname{Hom}_{R}(-,-)$.

(2) If the exact sequence $0 \rightarrow M^{\prime} \rightarrow M \rightarrow M^{\prime \prime} \rightarrow 0$ of $R$-modules is $\operatorname{Hom}_{R}(D,-)$ exact for all Ding projective $R$-modules $D$, then by part (2) of [14, Theorem 8.2.3] there is a long exact sequence

$\cdots \rightarrow \operatorname{Dext}_{R}^{i}(M,-) \rightarrow \operatorname{Dext}_{R}^{i}\left(M^{\prime},-\right) \rightarrow \operatorname{Dext}_{R}^{i+1}\left(M^{\prime \prime},-\right) \rightarrow \cdots$.

(3) If the exact sequence $0 \rightarrow N^{\prime} \rightarrow N \rightarrow N^{\prime \prime} \rightarrow 0$ of $R$-modules is $\operatorname{Hom}_{R}(-, C)$ exact for all Ding injective $R$-modules $C$, then by part (1) of [14, Theorem 8.2.5] there is a long exact sequence

$$
\cdots \rightarrow \operatorname{Dext}_{R}^{i}(-, N) \rightarrow \operatorname{Dext}_{R}^{i}\left(-, N^{\prime \prime}\right) \rightarrow \operatorname{Dext}_{R}^{i+1}\left(-, N^{\prime}\right) \rightarrow \cdots
$$

(4) There are natural transformations

$$
\operatorname{Dext}_{R}^{i}(-,-) \longrightarrow \operatorname{Ext}_{R}^{i}(-,-)
$$

which are also natural in the long exact sequences as in (2) and (3) above.

Proposition 3.8. Assume that $R$ is a Ding-Chen ring, $X$ is an $R$-module, and let $m$ be a non-negative integer. Then the following statements are equivalent:

(1) $1 . \operatorname{Dpd}_{R}(X) \leq m$. 
(2) For any exact sequence $0 \rightarrow K_{m} \rightarrow P_{m-1} \rightarrow \cdots \rightarrow P_{0} \rightarrow X \rightarrow 0$ with each $P_{i}$ projective, $K_{m}$ is Ding projective.

(3) $\operatorname{Ext}_{R}^{m+i}(X, F)=0$ for all flat $R$-modules $F$, and all $i \geq 1$.

(4) $\operatorname{Ext}_{R}^{m+i}(X, L)=0$ for all $L \in \mathcal{W}$, and all $i \geq 1$.

(5) $\operatorname{Dext}_{R}^{m+i}(X, Y)=0$ for all $R$-modules $Y$, and all $i \geq 1$.

(6) $\operatorname{Dext}_{R}^{m+1}(X, Y)=0$ for all $R$-modules $Y$.

Proof. $(1) \Leftrightarrow(2) \Leftrightarrow(3)$ follows from [7, Lemma 3.4] and (4) $\Rightarrow(3)$ is trivial.

$(3) \Rightarrow(4)$ If $L \in \mathcal{W}$, then there is an exact sequence $0 \rightarrow F_{n} \rightarrow F_{n-1} \rightarrow$ $\cdots \rightarrow F_{0} \rightarrow L \rightarrow 0$ with $F_{0}, F_{1}, \ldots, F_{n}$ flat. Breaking this exact sequence into short exact ones, and applying $\operatorname{Hom}_{R}(X,-)$, we get $\operatorname{Ext}_{R}^{m+i}(X, L) \cong$ $\operatorname{Ext}_{R}^{m+i+n}\left(X, F_{n}\right)=0$ for all $i \geq 1$, as desired.

$(2) \Rightarrow(5)$ Let $\cdots \rightarrow D_{1} \rightarrow D_{0} \rightarrow X \rightarrow 0$ be a proper left Ding projective resolution of $X$ and $H_{m}=\operatorname{Ker}\left(D_{m-1} \rightarrow D_{m-2}\right)$. Then by [7, Proposition 2.10] and [1, Lemma 3.12], $H_{m}$ is Ding projective. So $0 \rightarrow H_{m} \rightarrow D_{m-1} \rightarrow \cdots \rightarrow$ $D_{1} \rightarrow D_{0} \rightarrow X \rightarrow 0$ be a proper left Ding projective resolution of $X$ and thus $\operatorname{Dext}_{R}^{m+i}(X, Y)=0$ for all $R$-modules $Y$, and all $i \geq 1$.

$(5) \Rightarrow(6)$ It is trivial.

$(6) \Rightarrow(1)$ Let $\cdots \rightarrow D_{1} \rightarrow D_{0} \rightarrow X \rightarrow 0$ be a proper left Ding projective resolution of $X$ and $K=\operatorname{Ker}\left(D_{m} \rightarrow D_{m-1}\right)$. Then (6) implies that $\operatorname{Hom}_{R}\left(D_{m}, Y\right) \rightarrow \operatorname{Hom}_{R}(K, Y) \rightarrow 0$ is exact for all $R$-modules $Y$. So by setting $Y=K$, we see that $0 \rightarrow K \rightarrow D_{m} \rightarrow H_{m} \rightarrow 0$ is split exact, where $H_{m}=\operatorname{Ker}\left(D_{m-1} \rightarrow D_{m-2}\right)$, and so $K$ and $H_{m}$ are Ding projective by [7, Proposition 2.10], as desired.

With a dual proof we get the following.

Proposition 3.9. Assume that $R$ is a Ding-Chen ring, $Y$ is an $R$-module, and let $m$ be a non-negative integer. Then the following statements are equivalent:

(1) $\operatorname{l.Did}_{R}(Y) \leq m$.

(2) For any exact sequence $0 \rightarrow Y \rightarrow I^{0} \rightarrow \cdots \rightarrow I^{m-1} \rightarrow K^{m} \rightarrow 0$ with each $I^{i}$ injective, $K^{m}$ is Ding injective.

(3) $\operatorname{Ext}_{R}^{m+i}(E, Y)=0$ for all FP-injective $R$-modules $E$, and all $i \geq 1$.

(4) $\operatorname{Ext}_{R}^{m+i}(L, Y)=0$ for all $L \in \mathcal{W}$, and all $i \geq 1$.

(5) $\operatorname{Dext}_{R}^{m+i}(X, Y)=0$ for all $R$-modules $X$, and all $i \geq 1$.

(6) $\operatorname{Dext}_{R}^{m+1}(X, Y)=0$ for all $R$-modules $X$.

Definition 3.10. Let $R$ be a Ding-Chen ring. Then the global Ding projective dimension of the category of left $R$-modules, denoted $\operatorname{gl} \operatorname{lopd}(R)$, is defined by

$$
\text { gl 1.Dpd }(R)=\sup \left\{1 . \operatorname{Dpd}_{R}(M) \mid M \text { is any } R \text {-module }\right\} \text {. }
$$

Dually, the global Ding injective dimension of the category of left $R$-modules, denoted $\operatorname{gl} 1 . \operatorname{Did}(R)$, is defined by

$$
\text { gl l.Did }(R)=\sup \left\{1 \cdot \operatorname{Did}_{R}(M) \mid M \text { is any } R \text {-module }\right\} \text {. }
$$


Now we get the following result.

Theorem 3.11. Assume that $R$ is a Ding-Chen ring. Then the following statements are equivalent for some non-negative integer $m$.

(1) $\mathrm{gl}$ l.Dpd $(R) \leq m$.

(2) $\operatorname{gl} \operatorname{l.Did}(R) \leq m$.

(3) $\operatorname{Dext}_{R}^{m+i}(X, Y)=0$ for all $R$-modules $X, Y$, and all $i \geq 1$.

(4) $\operatorname{Dext}_{R}^{m+1}(X, Y)=0$ for all $R$-modules $X$ and $Y$.

Proof. This easily follows from Propositions 3.8 and 3.9 above.

Corollary 3.12. Assume that $R$ is a Ding-Chen ring. Then any submodule of a Ding projective $R$-module is Ding projective if and only if any quotient of a Ding injective $R$-module is Ding injective.

Proof. This is an immediate consequence of Theorem 3.11 by setting $m=$ 1.

Lemma 3.13. Assume that $R$ is a Ding-Chen ring, $X$ is an $R$-module, and let $\mathbf{D}=\cdots \rightarrow D_{1} \rightarrow D_{0} \rightarrow X \rightarrow 0$ be a proper left Ding projective resolution of $X$. Then $G \otimes_{R} \mathbf{D}$ is exact for all Gorenstein flat right $R$-modules $G$.

Proof. We note that $\cdots \rightarrow G \otimes_{R} D_{1} \rightarrow G \otimes_{R} D_{0} \rightarrow G \otimes_{R} X \rightarrow 0$ is exact if and only if $0 \rightarrow\left(G \otimes_{R} X\right)^{+} \rightarrow\left(G \otimes_{R} D_{0}\right)^{+} \rightarrow\left(G \otimes_{R} D_{1}\right)^{+} \rightarrow \cdots$ is exact. But the latter is equivalent to the sequence $0 \rightarrow \operatorname{Hom}_{R}\left(X, G^{+}\right) \rightarrow \operatorname{Hom}_{R}\left(D_{0}, G^{+}\right) \rightarrow$ $\operatorname{Hom}_{R}\left(D_{1}, G^{+}\right) \rightarrow \cdots$ which is exact by $[24$, Lemma 2.8] and Lemma 3.4.

This gives the following result.

Theorem 3.14. Let $R$ be an Ding-Chen ring. Then $-\otimes_{R}-$ is left balanced on $\mathcal{M}_{R} \times{ }_{R} \mathcal{M}$ by $\mathcal{D} \mathcal{P} \times \mathcal{D} \mathcal{P}$.

Remark 3.15. By Theorem 3.14 together with [14, Theorem 8.2.14], if $R$ is Ding-Chen, then we can compute left derived functors of $X \otimes_{R} Y$ using a proper left Ding projective resolution of $X$ or $Y$. We will denote these derived functors by $\operatorname{Dtor}_{i}^{R}(X, Y)$. Then it is easy to check the following properties of $\operatorname{Dtor}_{i}^{R}$ :

(1) $\operatorname{Dtor}_{0}^{R}(-,-)=-\otimes_{R}-$.

(2) $\operatorname{Dtor}_{i}^{R}(D,-)=0$ for all $i \geq 1$ and all Ding projective right $R$-modules $D$.

(3) $\operatorname{Dtor}_{i}^{R}(-, D)=0$ for all $i \geq 1$ and all Ding projective left $R$-modules $D$.

(4) If the exact sequence $0 \rightarrow M^{\prime} \rightarrow M \rightarrow M^{\prime \prime} \rightarrow 0$ of right $R$-modules is $\operatorname{Hom}_{R}(D,-)$ exact for all Ding projective right $R$-modules $D$, then by part (1) of [14, Theorem 8.2.3] there is a long exact sequence

$\cdots \rightarrow \operatorname{Dtor}_{i+1}^{R}\left(M^{\prime \prime},-\right) \rightarrow \operatorname{Dtor}_{i}^{R}\left(M^{\prime},-\right) \rightarrow \operatorname{Dtor}_{i}^{R}(M,-) \rightarrow \cdots$. 
(5) If the exact sequence $0 \rightarrow N^{\prime} \rightarrow N \rightarrow N^{\prime \prime} \rightarrow 0$ of left $R$-modules is $\operatorname{Hom}_{R}(D,-)$ exact for all Ding projective left $R$-modules $D$, then there is a long exact sequence

$\cdots \rightarrow \operatorname{Dtor}_{i+1}^{R}\left(-, N^{\prime \prime}\right) \rightarrow \operatorname{Dtor}_{i}^{R}\left(-, N^{\prime}\right) \rightarrow \operatorname{Dtor}_{i}^{R}(-, N) \rightarrow \cdots$.

(6) There are natural transformations

$$
\operatorname{Tor}_{i}^{R}(-,-) \longrightarrow \operatorname{Dtor}_{i}^{R}(-,-)
$$

which are also natural in the long exact sequences as in (4) and (5) above.

Naturally, we want to know what relationships there are between the new functor Dext (respectively, Dtor) and the classical one Ext (respectively, Tor), and we have the following result.

Proposition 3.16. Let $R$ be a Ding-Chen ring and $L$ an $R$-module. If $\operatorname{fd}_{R}(L)$ $<\infty$, then the following conclusions hold:

(1) The natural transformation $\operatorname{Dext}_{R}^{i}(L,-) \longrightarrow \operatorname{Ext}_{R}^{i}(L,-)$ is a natural isomorphism for all $i \geq 0$.

(2) The natural transformation $\operatorname{Dext}_{R}^{i}(-, L) \longrightarrow \operatorname{Ext}_{R}^{i}(-, L)$ is a natural isomorphism for all $i \geq 0$.

(3) The natural transformation $\operatorname{Tor}_{i}^{R}(-, L) \longrightarrow \operatorname{Dtor}_{i}^{R}(-, L)$ is a natural isomorphism for all $i \geq 0$.

Proof. (1) The assumption guarantees that $\operatorname{Ext}^{i}(L, C)=0$ for all $i \geq 1$ and all Ding injective $R$-modules $C$ by [17, Corollary 4.5] and [23, Theorem 3.4]. Now let $N \rightarrow C$ be a Ding injective preenvelope of $N$ and $K=C / N$. Then we have a commutative diagram

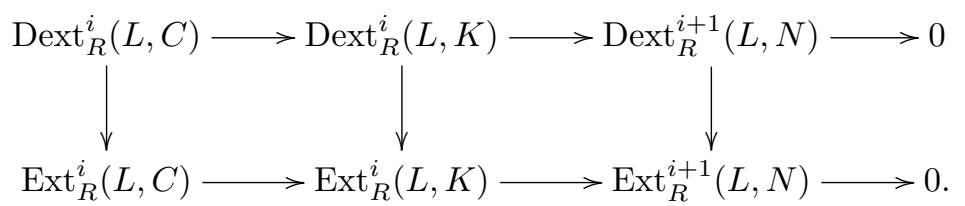

If $i=0$, then $\operatorname{Dext}_{R}^{0}(-,-)=\operatorname{Ext}_{R}^{0}(-,-)=\operatorname{Hom}_{R}(-,-)$ and so the first two maps are isomorphisms. Hence $\operatorname{Dext}_{R}^{1}(L, N) \longrightarrow \operatorname{Ext}_{R}^{1}(L, N)$ is an isomorphism. The result now follows by induction on $i$.

(2) The proof is similar to that of (1).

(3) We consider the exact sequence $0 \rightarrow H \rightarrow D \rightarrow N \rightarrow 0$ of right $R$ -

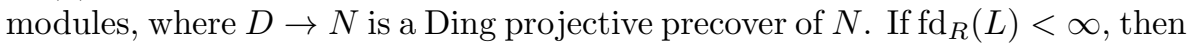
FP-id $_{R}(L)<\infty$ by Proposition 3.1, and so we have a commutative diagram

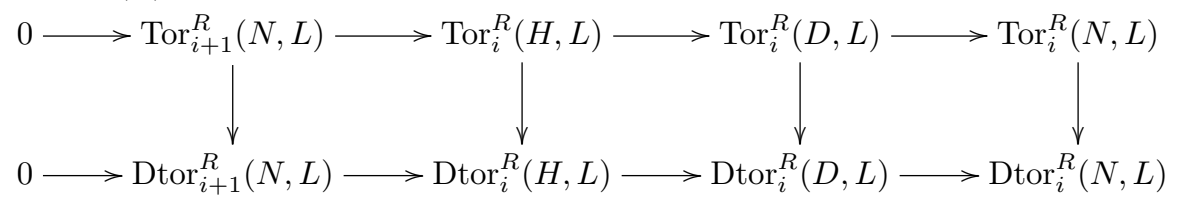


by $\left[6\right.$, Theorem 5] and $\left[7\right.$, Proposition 2.3]. But $\operatorname{Tor}_{0}^{R}(-,-)=\operatorname{Dtor}_{0}^{R}(-,-)=$ $-\otimes_{R}-$ and the result follows as in (1).

Corollary 3.17. Assume that $R$ is a Ding-Chen ring, $X$ is an $R$-module, and let $m$ be a non-negative integer. Then the following statements are equivalent.

(1) $1 . \operatorname{Dpd}_{R}(X) \leq m$.

(2) $\operatorname{Dext}_{R}^{m+i}(X, F)=0$ for all flat $R$-modules $F$, and all $i \geq 1$.

(3) $\operatorname{Dext}_{R}^{m+i}(X, L)=0$ for all $L \in \mathcal{W}$, and all $i \geq 1$.

Proof. Use Propositions 3.8 and 3.16.

Corollary 3.18. Assume that $R$ is a Ding-Chen ring, $Y$ is an $R$-module, and let $m$ be a non-negative integer. Then the following statements are equivalent:

(1) $\operatorname{l.Did}_{R}(Y) \leq m$.

(2) $\operatorname{Dext}_{R}^{m+i}(E, Y)=0$ for all FP-injective $R$-modules $E$, and all $i \geq 1$.

(3) $\operatorname{Dext}_{R}^{m+i}(L, Y)=0$ for all $L \in \mathcal{W}$, and all $i \geq 1$.

Proof. Use Propositions 3.9 and 3.16 .

We also have the following result.

Proposition 3.19. Let $R$ be a Ding-Chen ring and $N$ an $R$-module. Then

(1) If $M$ is an $R$-module, then $\operatorname{Dext}_{R}^{1}(M, N) \longrightarrow \operatorname{Ext}_{R}^{1}(M, N)$ is an injection.

(2) If $M$ is a right $R$-module, then $\operatorname{Tor}_{1}^{R}(M, N) \longrightarrow \operatorname{Dtor}_{1}^{R}(M, N)$ is a surjection.

Proof. (1) Let $N \rightarrow C$ be a Ding injective preenvelope of $N$ and $K=C / N$. Then we have the following diagram

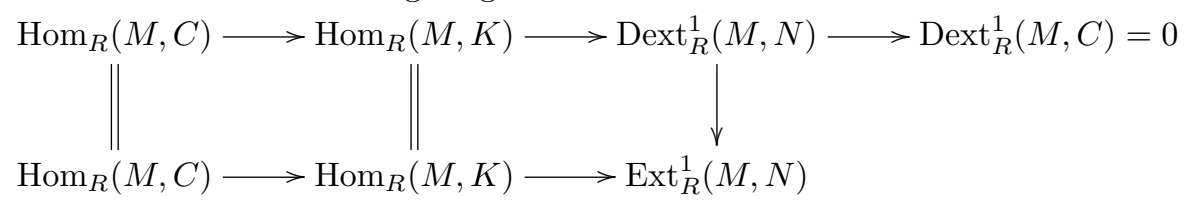

with exact rows. It is easy to see that $\operatorname{Dext}_{R}^{1}(M, N) \longrightarrow \operatorname{Ext}_{R}^{1}(M, N)$ is an injection.

(2) The proof is similar to that of (1).

If we consider the elements of $\operatorname{Ext}_{R}^{1}(M, N)$ as classes of short exact sequences $0 \rightarrow N \rightarrow L \rightarrow M \rightarrow 0$ of $R$-modules, then we get the following result.

Corollary 3.20. Let $R$ be a Ding-Chen ring. Then the following are equivalent for an exact sequence $0 \rightarrow N \rightarrow L \rightarrow M \rightarrow 0$ of $R$-modules.

(1) The sequence $0 \rightarrow N \rightarrow L \rightarrow M \rightarrow 0$ corresponds to an element of $\operatorname{Dext}_{R}^{1}(M, N) \subset \operatorname{Ext}_{R}^{1}(M, N)$.

(2) The sequence $\operatorname{Hom}_{R}(L, C) \rightarrow \operatorname{Hom}_{R}(N, C) \rightarrow 0$ is exact for all Ding injective $R$-modules $C$. 
(3) The sequence $\operatorname{Hom}_{R}(D, L) \rightarrow \operatorname{Hom}_{R}(D, M) \rightarrow 0$ is exact for all Ding projective $R$-modules $D$.

Proof. (1) $\Rightarrow(2)$ If $0 \rightarrow N \rightarrow L \rightarrow M \rightarrow 0$ corresponds to an element of $\operatorname{Dext}_{R}^{1}(M, N)$, then there is a commutative diagram

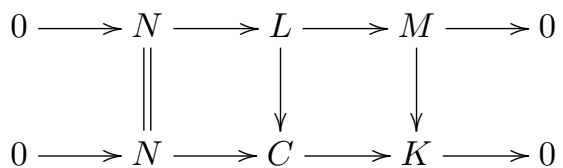

with exact rows such that $N \rightarrow C$ is a Ding injective preenvelope. But if $N \rightarrow C^{\prime}$ is a map with $C^{\prime}$ Ding injective, then $N \rightarrow C^{\prime}$ can be extended to $C$. But then $N \rightarrow C^{\prime}$ can be extended to $L$ and so the result follows.

$(2) \Rightarrow(1)$ Exactness of the sequence $\operatorname{Hom}_{R}(L, C) \rightarrow \operatorname{Hom}_{R}(N, C) \rightarrow 0$ for all Ding injective $R$-modules $C$ implies that we have the following diagram

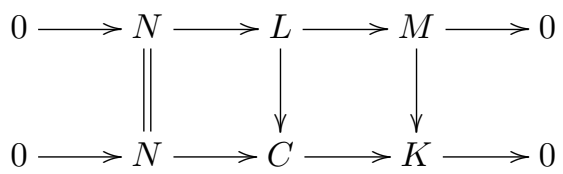

with exact rows, where $N \rightarrow C$ is a Ding injective preenvelope of $N$. This shows that the sequence $0 \rightarrow N \rightarrow L \rightarrow M \rightarrow 0$ corresponds to an element of $\operatorname{Dext}_{R}^{1}(M, N)$.

$(1) \Leftrightarrow(3)$ It follows by a dual argument.

We recall from [27, Definition 3.1.1] that an $R$-module $K$ is called cotorsion if $\operatorname{Ext}_{R}^{1}(F, K)=0$ for all flat $R$-modules $F$. Recall from [10] that an $R$-module $L$ is called Gorenstein cotorsion if $\operatorname{Ext}_{R}^{1}(G, L)=0$ for all Gorenstein flat $R$ modules $G$. Clearly, any Gorenstein cotorsion module is cotorsion.

Lemma 3.21. If $R$ is Ding-Chen, then an $R$-module $M$ is Gorenstein cotorsion if and only if it is cotorsion and has finite flat dimension.

Proof. $(\Rightarrow)$ Since $R$ is Ding-Chen, $\left({ }^{\perp} \mathcal{W}, \mathcal{W}\right)$ forms a complete cotorsion pair by $[8$, Theorem 3.8], where $\mathcal{W}$ denotes the class of all modules with finite flat dimension. Also we know that $\perp^{\mathcal{W}}$ is just the class of all Ding projective modules by [17, Corollary 4.6]. Now by [17, Proposition 3.11] and [17, Proposition 3.12] it follows that any Gorenstein cotorsion module has finite flat dimension.

$(\Leftarrow)$ Let $M$ be a cotorsion module with finite flat dimension. By [17, Lemma 4.8], there exists an exact sequence $0 \rightarrow M \rightarrow C \rightarrow F \rightarrow 0$ such that $C$ is Gorenstein cotorsion and $F$ Gorenstein flat. By the last paragraph, we see that $C$ has finite flat dimension. Therefore $F$ has finite flat dimension, which implies $F^{+}=\operatorname{Hom}_{\mathbb{Z}}(F, \mathbb{Q} / \mathbb{Z})$ has finite injective dimension. On the other hand, $F^{+}$is Ding injective by [24, Lemma 2.8]. Thus we get that $F^{+}$is injective by [24, Lemma 2.3], and so $F$ is flat by [14, Theorem 3.2.9]. Now we see that 
the sequence $0 \rightarrow M \rightarrow C \rightarrow F \rightarrow 0$ is split and so $M$ is a summand of $C$. Therefore $M$ is Gorenstein cotorsion.

It was shown in [15] that every module $X$ has a Gorenstein flat cover over right coherent rings. The existence of Gorenstein flat covers was also proved for modules over more general rings (see [28]). Thus we see that if $R$ is right coherent, then every $R$-module $X$ admits a proper left Gorenstein flat resolution.

Lemma 3.22. Assume that $R$ is a Ding-Chen ring, $M$ is an $R$-module, and let $\mathbf{G}=\cdots \rightarrow G_{1} \rightarrow G_{0} \rightarrow M \rightarrow 0$ be a proper left Gorenstein flat resolution of $M$. Then $H \otimes_{R} \mathbf{G}$ is exact for all Gorenstein flat right $R$-modules $H$.

Proof. From arguments above, there exists an exact sequence

$$
\mathbf{S}=0 \rightarrow L \rightarrow G \rightarrow M \rightarrow 0
$$

such that $G \rightarrow M$ is a Gorenstein flat cover of $M$, and so by Wakamutsu's lemma, we get that $L$ is Gorenstein cotorsion. Thus $L$ is cotorsion and $\operatorname{fd}_{R}(L)<$ $\infty$ by Lemma 3.21. Hence it suffices to show the exactness of $H \otimes_{R} \mathbf{S}$ whenever $H$ is a Gorenstein flat right $R$-module.

Now let $H$ be a Gorenstein flat right $R$-module. Note that the sequence

$$
0 \rightarrow H \otimes_{R} L \rightarrow H \otimes_{R} G \rightarrow H \otimes_{R} M \rightarrow 0
$$

is exact if and only if the sequence

$$
0 \rightarrow\left(H \otimes_{R} M\right)^{+} \rightarrow\left(H \otimes_{R} G\right)^{+} \rightarrow\left(H \otimes_{R} L\right)^{+} \rightarrow 0
$$

is exact. But the latter is equivalent to the sequence

$$
0 \rightarrow \operatorname{Hom}_{R}\left(M, H^{+}\right) \rightarrow \operatorname{Hom}_{R}\left(G, H^{+}\right) \rightarrow \operatorname{Hom}_{R}\left(L, H^{+}\right) \rightarrow 0 .
$$

Since $H^{+}$is Ding injective, then the result follows by using the same way as in Lemma 3.4 .

It was proved by Enochs and Jenda [14, Theorem 12.2.2] that the functor - $\otimes_{R}$ - is left balanced on $\mathcal{M}_{R} \times{ }_{R} \mathcal{M}$ by $\mathcal{G} \mathcal{F} \times \mathcal{G F}$ when $R$ is Gorenstein. Recently, this result was generalized by Holm [19, Theorem 4.8]. Here, we also have its another generalization as follows.

Theorem 3.23. Let $R$ be a Ding-Chen ring. Then $-\otimes_{R}-$ is left balanced on $\mathcal{M}_{R} \times{ }_{R} \mathcal{M}$ by $\mathcal{G F} \times \mathcal{G F}$.

It is known that every module over Ding-Chen rings has a Gorenstein flat preenvelope [24, Theorem 5.3]. Thus we see that if $R$ is Ding-Chen, then every $R$-module $M$ admits a proper right Gorenstein flat resolution.

Lemma 3.24. Let $R$ be a Ding-Chen ring, $X$ and $Y$ be $R$-modules. Then

(1) If $\mathbf{H}=0 \rightarrow X \rightarrow H^{0} \rightarrow H^{1} \rightarrow \cdots$ is a proper right Gorenstein flat resolution of $X$, then $B \otimes_{R} \mathbf{H}$ is exact for all Ding injective right $R$-modules $B$. 
(2) If $\mathbf{C}=0 \rightarrow Y \rightarrow C^{0} \rightarrow C^{1} \rightarrow \cdots$ is a proper right Ding injective resolution of $Y$, then $G \otimes_{R} \mathbf{C}$ is exact for all Gorenstein flat right $R$-modules $G$.

Proof. (1) We note that $0 \rightarrow B \otimes_{R} X \rightarrow B \otimes_{R} H^{0} \rightarrow B \otimes_{R} H^{1} \rightarrow \cdots$ is exact if and only if $\cdots \rightarrow\left(B \otimes_{R} H^{1}\right)^{+} \rightarrow\left(B \otimes_{R} H^{0}\right)^{+} \rightarrow\left(B \otimes_{R} X\right)^{+} \rightarrow 0$ is exact. But the latter is equivalent to the sequence $\cdots \rightarrow \operatorname{Hom}_{R}\left(H^{1}, B^{+}\right) \rightarrow$ $\operatorname{Hom}_{R}\left(H^{0}, B^{+}\right) \rightarrow \operatorname{Hom}_{R}\left(X, B^{+}\right) \rightarrow 0$ which is exact by [6, Proposition 12].

(2) The proof is similar to that of (1).

This gives the following result.

Theorem 3.25. Let $R$ be a Ding-Chen ring. Then $-\otimes_{R}-$ is right balanced on $\mathcal{M}_{R} \times{ }_{R} \mathcal{M}$ by $\mathcal{G} \mathcal{F} \times \mathcal{D} \mathcal{I}$.

Acknowledgements. The author likes to thank the referee for careful reading and valuable comments that improved the present article.

\section{References}

[1] M. Auslander and M. Bridger, Stable Module Theory, Memoirs of the American Mathematical Society, No. 94 American Mathematical Society, Providence, R.I. 1969.

[2] D. Bennis, Rings over which the class of Gorenstein flat modules is closed under extensions, Comm. Algebra, 37 (2009), no. 3, 855-868.

[3] R. F. Damiano, Coflat rings and modules, Pacific J. Math. 81 (1979), no. 2, 349-369.

[4] N. Q. Ding and J. L. Chen, The flat dimensions of injective modules, Manuscripta Math. 78 (1993), no. 2, 165-177.

[5] - The homological dimensions of simple modules, Bull. Aust. Math. Soc. 48 (1993), no. 2, 265-274.

[6] Coherent rings with finite self-FP-injective dimension, Comm. Algebra 24 (1996), no. 9, 2963-2980.

[7] N. Q. Ding, Y. L. Li, and L. X. Mao, Strongly Gorenstein flat modules, J. Aust. Math. Soc. 86 (2009), no. 3, 323-338.

[8] N. Q. Ding and L. X. Mao, Relative FP-projective modules, Comm. Algebra 33 (2005), no. 5, 1587-1602.

[9] E. E. Enochs, Injective and flat covers, envelopes and resolvents, Israel J. Math. 39 (1981), no. 3, 189-209.

[10] E. E. Enochs, S. Estrada, and B. Torrecillas, Gorenstein flat covers and gorenstein cotorsion modules over integral group rings, Algebr. Represent. Theory 8 (2005), no. 4, $525-539$.

[11] E. E. Enochs and Z. Y. Huang, Injective envelopes and (Gorenstein) flat covers, in press.

[12] E. E. Enochs and O. M. G. Jenda, Balanced functors applied to modules, J. Algebra 92 (1985), 303-310.

[13] _ Gorenstein balance of Hom and tensor, Tsukuba J. Math. 19 (1995), no. 1, $1-13$.

[14] _ Relative Homological Algebra, De Gruyter Expositions in Mathematics no. 30, Walter De Gruyter, New York, 2000

[15] E. E. Enochs, O. M. G. Jenda, and J. A. López-Ramos, The existence of Gorenstein flat covers, Math. Scand. 94 (2004), no. 1, 46-62.

[16] E. E. Enochs, O. M. G. Jenda, and B. Torrecillas, Gorenstein flat modules, Nanjing Daxue Xuebao Shuxue Bannian Kan, 10 (1993), no. 1, 1-9. 
[17] J. Gillespie, Model structures on modules over Ding-Chen rings, Homology, Homotopy Appl. 12 (2010), no. 1, 61-73.

[18] H. Holm, Gorenstein homological dimensions, J. Pure Appl. Algebra 189 (2004), no. 1-3, 167-193.

[19] — Gorenstein derived functors, Proc. Amer. Math. Soc. 132 (2004), no. 7, 19131923.

[20] M. Hovey, Cotorsion pairs, model category structures, and representation theory, Math. Z. 241 (2002), no. 3, 553-592.

[21] Y. Iwanaga, On rings with finite self-injective dimension, Comm. Algebra 7 (1979), no. $4,393-414$

[22] $1,107-113$.

[23] L. X. Mao and N. Q. Ding, Envelopes and covers by modules of finite FP-injective and flat dimensions, Comm. Algebra 35 (2007), no. 3, 833-849.

[24] , Gorenstein FP-injective and Gorenstein flat modules, J. Algebra Appl. 7 (2008), no. 4, 491-506.

[25] W. L. Song and Z. Y. Huang, Gorenstein flatness and injectivity over Gorenstein rings, Sci. China Ser. A 51 (2008), no. 2, 215-218.

[26] B. Stenström, Coherent rings and FP-injective modules, J. London Math. Soc. 2 (1970), no. 2, 323-329.

[27] J. Z. Xu, Flat Covers of Modules, Lecture Notes in Math, 1634, 1996.

[28] G. Yang and Z. K. Liu, Gorenstein flat covers over GF-closed rings, Comm. Algebra, to appear.

School of Mathematics

Physics And Software EngineERING

LANZHOU JIAOTONG UNIVERSITY

LANZhou 730070, P. R. China

E-mail address: yanggang@mail.lzjtu.cn 